\title{
Diagnóstico Situacional PEST-LA para Identificar los Problemas Turísticos de la Provincia de Cotopaxi, Ecuador
}

\author{
Milton Alberto Sampedro Arrieta, $\mathrm{MsC}$ \\ Manuel Antonio Abarca Zaquinaula, MsC \\ Diana Karina Vinueza Morales, MsC \\ Docentes Tiempo Completo de la Carrera de Turismo \\ Universidad Técnica de Cotopaxi (UTC), Ecuador \\ Kimberley Dayanna Rivas Bonilla, \\ Estudiante de la Carrera de Turismo \\ Universidad Técnica de Cotopaxi (UTC), Ecuador
}

Doi:10.19044/esj.2020.v16n17p29 ～URL:http://dx.doi.org/10.19044/esj.2020.v16n17p29

\section{Resumen}

El turismo es una actividad económica en crecimiento en el mundo, que genera múltiples impactos positivos y negativos, para su crecimiento necesita el buen funcionamiento de los factores del macro-entorno, por lo que, el presente trabajo modificó la matriz PEST-LA y analizó los factores; político, económico, social-cultural, tecnológico, legal y ambiental de los cantones; La Mana, Salcedo, Saquisilí, Latacunga y Pujilí. Para su análisis se trabajó con 116 elementos y cada elemento analizado se subdividió en variables que fueron valoradas y calificadas con base en las evidencias encontradas, a través de información segundaria e información primaria (entrevistas a actores claves), obteniendo así; 74 elementos valorados como negativos (catalogados como problemas turísticos) y 42 elementos valorados como positivos, correspondientes al diagnóstico situacional de los problemas que no están permitiendo el desarrollo turístico en la provincia de Cotopaxi.

Palabras clave: Diagnóstico turístico, Matriz PEST-LA, Macro-entorno, Sistema Turístico 


\title{
Situational Diagnosis to Identify Tourist Problems of the Province of Cotopaxi, Ecuador
}

\author{
Milton Alberto Sampedro Arrieta, $\mathrm{MsC}$ \\ Manuel Antonio Abarca Zaquinaula, MsC \\ Diana Karina Vinueza Morales, $M s C$ \\ Docentes Tiempo Completo de la Carrera de Turismo \\ Universidad Técnica de Cotopaxi (UTC), Ecuador \\ Kimberley Dayanna Rivas Bonilla, \\ Estudiante de la Carrera de Turismo \\ Universidad Técnica de Cotopaxi (UTC), Ecuador
}

\begin{abstract}
Tourism is a growing economic activity in the world, which generates multiple positive and negative impacts, for its growth it needs the proper functioning of the macro-environment factors, therefore, this work modified the PEST-LA matrix and analyzed the facts; political, economic, socialcultural, technological, legal and environmental of the cantons; La Mana, Salcedo, Saquisilí, Latacunga and Pujilí. For its analysis we worked with 116 elements and each element analyzed was subdivided into variables that were assessed and qualified based on the evidence found, through secondary information and primary information (interviews with key actors), thus obtaining; 78 elements valued as negative (cataloged as tourist problems) and 38 elements valued as positive, corresponding to the situational diagnosis of problems that are not allowing tourism development in the province of Cotopaxi.
\end{abstract}

Keywords: Tourism diagnosis, PEST-LA Matrix, Macro-environment, Tourist System

\section{Introduction}

El turismo es una de las principales actividades económicas en el mundo, generando importantes movimientos monetarios, permitiendo la inversión nacional y extranjera, creando empleos directos e indirectos, contribuyendo al crecimiento económico y al progreso social de los países en vías de desarrollo, pues favorece la realización de actividades que atienden la demanda de los turistas como; hospedaje, alimentación, transporte, diversión, conocimiento de tradiciones, entre otras. Además, impulsa la mejora continua 
de infraestructura regional mediante la construcción de urbanizaciones y vías de comunicación aérea, terrestre o marítima (Dalles, 2012). El turismo apunta a consolidarse como una de las principales ramas de actividad económica a nivel mundial, con muchas posibilidades de crecimiento.

El turismo mundial crece un 7 \%. En 2017, se registró el mayor crecimiento turístico desde 2010, impulsado por un aumento de las llegadas a Europa, que ha visto un incremento del 8\%, pero también por la recuperación de regiones de Oriente Medio, con una subida del 5\%. En total, el año anterior registró 1.322 millones de viajeros internacionales, un 7\% más que el ejercicio anterior (OMT, 2008, pág. 23).

De modo que los impactos que se derivan de esta actividad son de naturaleza diversa, donde el turista pasa a ser un gran consumidor de bienes y servicios, su presencia dinamiza diversos sectores de la actividad económica, genera riqueza y empleo e introduce nuevos hábitos, modos de vida y usos del espacio público urbano. Por lo tanto, tiene un amplio abanico de efectos positivos y negativos sobre el cuerpo social de un territorio o destino turístico (Troitiño Vinueza \& Troitiño Torralba, 2016). La actividad turística es considerada también como fuente de desarrollo, en distintos ámbitos y en distintitos destinos turísticos de una nación, región o pueblo donde se desarrolle el turismo, de manera que ayuda a combatir la pobreza y fomenta el crecimiento creando nuevas oportunidades de empleos y emprendimientos mejorando la calidad de vida de los residentes del lugar. El turismo es una industria dinámica que moviliza personas por todo el planeta, influye en los ámbitos ambiental, económico, social y cultural, por esta razón diversos países han decidido impulsar el turismo como estrategia de desarrollo local (Rodriguez \& Granados, 2016). Además, al analizar los ingresos del turismo internacional por habitante, se comprueba que la UNASUR en el período 1995-2013 tuvo un promedio de US \$82; y para el año 2013 fue de US\$ 138. Mientras que Ecuador, en el período 1995-2013 tuvo un promedio de US\$ 41 y para el año 2013 fue de US\$ 80. Los ingresos del turismo internacional por habitante en Ecuador han seguido una tendencia creciente (Castillo, Vásquez, \& Martínez, 2015).

Tabla 1. Turismo Internacional: Turistas por habitante e ingresos por habitante.

UNASUR (1995- 2013)

\begin{tabular}{lcccc}
\hline País & \multicolumn{2}{c}{ Turista/ 1000 habitantes } & \multicolumn{2}{l}{$\begin{array}{l}\text { Ingreso/ } \\
\mathbf{2 0 0 7 )}\end{array}$} \\
\cline { 2 - 6 } & $\mathbf{1 9 9 5 - \mathbf { 2 0 1 3 }}$ & $\mathbf{2 0 1 3}$ & $\mathbf{1 9 9 5} \mathbf{- 2 0 1 3}$ & $\mathbf{2 0 1 3}$ \\
\hline Argentina & 97 & 131 & 97 & 128 \\
Bolivia & 54 & 54 & 29 & 61 \\
Brasil & 25 & 28 & 19 & 34 \\
Chile & 136 & 203 & 114 & 181
\end{tabular}




\begin{tabular}{lcccc} 
Colombia & 30 & 48 & 50 & 101 \\
Ecuador & 59 & 87 & 41 & 80 \\
Guyana * & 146 & 233 & 90 & 101 \\
Paraguay & 69 & 94 & 26 & 46 \\
Perú & 54 & 104 & 58 & 128 \\
Suriname & 247 & 467 & 118 & 172 \\
Uruguay & 615 & 787 & 316 & 590 \\
Venezuela ** & 25 & 33 & 30 & 30 \\
UNASUR & 130 & 189 & 82 & 138 \\
\hline NOTA: * Número de turistas al 2012 & * Ingresos de turismo Internacional al 2012 \\
\hline
\end{tabular}

Fuente: Castillo, Vásquez, \& Martínez (2015)

Ecuador es un país que cuenta con una gran diversidad natural y cultural en sus cuatro regiones que han dado lugar a la proliferación de cientos de especies de flora y fauna. Cuenta con alrededor de 1640 clases de pájaros. Las especies de mariposas bordean las 4.500, los reptiles 345, los anfibios 358 y los mamíferos 258, entre otras. No en vano el Ecuador está considerado como uno de los 17 países donde está concentrada la mayor biodiversidad del planeta, siendo además el mayor país con biodiversidad por $\mathrm{km} 2$ del mundo (Ramirez, 2013). El turismo en el Ecuador al igual que a nivel mundial se encuentra en crecimiento es así que, en el periodo 2018 se registró un incremento de visitantes, entre Enero y Noviembre de 2018 se registraron entradas de extranjeros al Ecuador de 2.204.435, con un crecimiento del 53,4\% en comparación al 2017. En este año llegaron al país 311.522 personas de Estados Unidos, la cifra subió al 41,8\% de aumento frente al 2017. También se incrementó el número de turistas de Canadá 30.496 con un aumento del $20,0 \%$ en comparación al año pasado, mientras que los provenientes desde Alemania crecieron 5,9\%, desde Francia $20 \%$ y Reino Unido 10,0\% (MINTUR, 2018).

Tabla 2. Crecimiento turístico de Ecuador 2018

\begin{tabular}{lccc}
\hline País & $\underline{\mathbf{2 0 1 7}}$ & $\underline{\mathbf{2 0 1 8}}$ & $\underline{\text { \% Crecimiento }}$ \\
Estados unidos & 219.16 & 311.522 & $41.80 \%$ \\
Canadá & 25.405 & 30.496 & $20.0 \%$ \\
Alemania & 30.744 & 32.546 & $5.9 \%$ \\
Reino Unido & 23.758 & 26.129 & $10.0 \%$ \\
\hline
\end{tabular}

Fuente: (Ministerio de Turismo, 2018)

El ingreso de divisas en el mes de enero y junio por concepto de turismo, registrado en la Balanza turística ascendió a 1.156,9 millones de dólares en el 2018, es decir, 49,6\% de incremento con respecto al ingreso registrado en el 2017. Esta cifra significa que ingresaron al país más divisas por concepto de gasto de turismo receptor, que las que salieron por gasto de 
un turismo emisor. Por esta razón el turismo en el año 2018 se convirtió en la tercera fuente de ingreso después del banano y el camarón. En el tercer trimestre del año 2018 se registraron 513.223 empleos relacionados con el sector turístico, lo que revela un crecimiento del 3,0\% respecto al año 2017. Esta cifra representa el 6,5\% del total de empleados en la economía del país, por lo que este aporte ubica a la actividad de alojamiento y servicios de comida entre las cinco industrias económicas de mayor contribución al empleo nacional. Lo que lo convierte en uno de los sectores que mayores empleos genera en Ecuador (MINTUR, 2018).

Tabla 3. Número de establecimientos registrados en el MINTUR

\begin{tabular}{lccccc}
\hline ACTIVIDAD & $\mathbf{2 0 1 1}$ & $\underline{\mathbf{2 0 1 2}}$ & $\underline{\mathbf{2 0 1 3}}$ & $\mathbf{2 0 1 4}$ & $\mathbf{2 0 1 5}$ \\
\hline Alojamiento & 4.032 & 4.334 & 4.672 & 5.189 & 5.488 \\
Servicio de alimentos y bebidas & 12.496 & 13.261 & 14.057 & 15.741 & 17.325 \\
Otros & 2.150 & 2.134 & 2.340 & 2.525 & 2.859 \\
TOTAL & $\mathbf{1 8 . 6 7 8}$ & $\mathbf{1 9 . 7 2 9}$ & $\mathbf{2 1 . 0 6 9}$ & $\mathbf{2 3 . 4 5 5}$ & $\mathbf{2 5 . 6 7 2}$ \\
\hline
\end{tabular}

Fuente: (CFN, 2017)

Nota: Incluye actividades de operación, intermediación y centros de recreación

El Turismo en Ecuador es un sector con proyección en el corto y largo plazo, que tendrá resultados tangibles en la generación de riqueza, empleo, buen vivir e impacto económico. Al tratarse un destino no maduro, el país puede "aprender" de las experiencias llevadas a cabo en otros lugares y tratar de poner en marcha tipologías de turismo e implementar productos turísticos más sostenibles y respetuosos con el medio ambiente (Castillo, Martínez, \& Vázquez, 2015). El desarrollo del turismo en el Ecuador a pesar de tener un enfoque hacia la igualdad y equidad social presenta problemas especialmente de desigualdad y desequilibrio ya que en gran parte del manejo de los recursos se encuentran en manos de empresas privadas o a su vez de grandes empresarios, aunque no se puede dejar de lado el tema de que en la misma comunidad existen personas que velan por intereses personales y no por el bienestar comunitario, como es el caso de "Los Siona en la Reserva Faunística del Cuyabeno, donde según (Prieto, 2011) el turismo que se ha desarrollado en la zona no es sustentable enfatizando en la necesidad de involucrar a la comunidad en los esfuerzos de protección ".

En el Ecuador persisten los problemas en el sector turístico, a pesar de contar con una gran diversidad natural, paisajística y multicultural, que no permiten aprovechar sosteniblemente los productos y atractivos turísticos existentes (Ministerio de Turismo, 2015). Entre los problemas identificados, se encuentran los siguientes; limitada evaluación de la competencia turística, baja promoción a gran escala y relaciones públicas limitadas con la industria turística y otros actores relacionados. Información del mercado turístico para la toma de decisiones limitado, pérdida de participación en el mercado turístico global, falta de estrategias para una acertada promulgación de la 
información, promoción y comercialización del turismo ecuatoriano, alto margen de desconocimiento de la oferta turística por nichos. Así también, en lo referente a una estrategia de promoción dinamizadora a la que se refiere el estudio realizado por CEPAL, se determina que el Ecuador debe focalizarse en experiencias, mercados y segmentos específicos de tal manera que se pueda generar un plan de acciones de alto impacto (Ministerio de Turismo, 2015).

El presente estudio busca analizar la problemática turística de la Provincia de Cotopaxi, especialmente el ambiente externo en toda su magnitud, identificando la multi-causalidad que no permite el desarrollo turístico en todo su esplendor.

\section{Metodología}

El presente estudio es transversal, de enfoque cualitativo con alcance exploratorio, empleando la metodología PEST-LA con la técnica de la entrevista y revisión de información secundaria, para diagnosticar situacionalmente los problemas turísticos existentes en la provincia de Cotopaxi. El diagnóstico es situacional porque trata fundamentalmente sobre la relación entre actores sociales y sus acciones; se centra en identificar las posibles determinaciones y relaciones causales que inciden en la producción de los fenómenos sociales y en delimitar las posibilidades de acción (Arteaga Basurto, C \& M.V. Gonzáles Montaño, 2001), y es turístico por que analiza el proceso de planificación donde se establece y evalúa la situación de un destino turístico en un momento determinado. En otras palabras, el diagnóstico consiste en una suerte de corte temporal que establece una diferencia entre la situación del turismo antes y los resultados obtenidos después de la aplicación de acciones o estrategias de planificación turística en un destino (Quijano, 2009).

\section{Método PEST-LA}

La matriz PEST-LA es una herramienta que permite analizar los diferentes aspectos, en los ámbitos; político, económico, social-cultural, tecnológico, legal y ambiental de un determinado sector. "La matriz PEST-LA esta específicamente diseñada para analizar los cambios sufridos en el entorno que afectarán. De este modo analizar cómo cambian estos factores" (Marketing, 2000, pág. 46). Se empleó este método para analizar los elementos que intervienen en el funcionamiento de los factores; político, económico, social-cultural, tecnológico, legal y ambiental, que de alguna manera están obstaculizando el desarrollo del turismo sostenible en la provincia de Cotopaxi, teniendo presente que el turismo es una actividad que depende del buen funcionamiento de todos los factores antes mencionados para su desarrollo. 
Tabla 1. Matriz PEST-LA

\begin{tabular}{lcccc}
\hline A. Factor & $\begin{array}{c}\text { B. Elementos } \\
\text { analizados }\end{array}$ & $\begin{array}{c}\text { C. Impacto } \\
\text { Positivo/Negativo }\end{array}$ & $\begin{array}{c}\text { D. Valoración } \\
\text { del Impacto }\end{array}$ & $\begin{array}{c}\text { E. Calificación } \\
1-3\end{array}$ \\
\hline $\begin{array}{l}\text { Político } \\
\text { Económico }\end{array}$ & & & \\
Social-cultural & & & \\
Tecnológico & & & \\
Legal & & & \\
Ambiental & & & \\
\hline
\end{tabular}

Fuente: Adaptado de (Cervantes, 2015)

A.- Se colocaron los factores (políticos, económicos, sociales, tecnológicos, legales y ambientales). B.- Se ubicaron los elementos analizados, identificados a través de las visitas in situ y las entrevistas realizadas a los actores claves, permitiendo de esta manera conocer los aspectos positivos y negativos encontrados en la investigación C.- Se midió el grado de impacto (positivo o negativo) D.- Se colocó el (nivel de impacto) de los elementos positivos o negativos. E.- Se colocó la calificación dependiendo del nivel del impacto.

Con la matriz PEST-LA se analizaron los factores determinantes del entorno turístico de la provincia de Cotopaxi, en los ámbitos político, económico, social-cultural, tecnológico, legal y ambiental, identificando los problemas turísticos existentes en los cantones; Latacunga, Pujilí, Saquisilí, Salcedo y La Mana. En el factor político se analizó y evaluó todos los elementos con tendencias políticas y el posicionamiento de los líderes que pueden influir dentro de su segmento, evaluando el impacto de todo cambio político o legislativo que pudo afectar o favorecer al sistema turístico de la provincia. En el factor económico se analizó y evaluaron los aspectos que se llevaron a cabo durante el desarrollo de la dinámica económica dentro del área de estudio. En el factor social-cultural se analizó las fuerzas que actúan dentro de la sociedad, que pueden alterar las actitudes, intereses y opiniones de la gente, así como también favorecer su entorno social con respecto al cambio de su estilo de vida. En el factor tecnológico se analizó y evaluó variables asociadas a la tecnología como fuerza impulsora de los negocios, mejora de calidad y reducción de los tiempos para mercadear productos y servicios dentro del área de estudio. En el factor legal se evaluó y analizó el cumplimiento de la normativa ambiental y turística. En el factor ambiental se analizó y evaluó los aspectos asociados a las leyes orientadas a proteger el medio ambiente y el nivel de conciencia ambiental de los habitantes y turistas dentro del área de estudio.

Cada elemento estudiado estuvo integrado por un conjunto de variables identificadas por el equipo investigador con base en la información primaria y secundaria obtenida del área de estudio. En cada variable fue necesario 
identificar los aspectos que tendrían más peso en el entorno futuro y otros que serían menos decisivos e irrelevantes dentro del sistema turístico de la provincia. Se identificó como impacto negativo a los aspectos que generan inconformidad, limitando el desarrollo local enfocado al turismo, mientras que el impacto positivo a todos los aspectos que contribuyen al desarrollo local enfocado al turismo. Para la calificación de los factores se tomó en cuenta la información de las variables del diagnóstico situacional del área de estudio y el criterio profesional del equipo investigador conjuntamente con los aspectos que tienen relación con el desarrollo del turismo en la provincia de Cotopaxi. La valoración y calificación se la realizó de la siguiente manera:

- Impacto Negativo: de 0 a 2 variables que no cumple el elemento analizado.

- Impacto Positivo: de 3 a 5 variables que cumple el elemento analizado

Para la valoración del impacto consideró el número de variables que cumple o no cumple el elemento analizado dentro de la matriz, su valoración se la realizó de la siguiente manera:

- Impacto alto: 4 - 5 variables de cumplimiento.

- Impacto medio: 2 - 3 variables de cumplimiento

- Impacto bajo: 0 - 1 variable de cumplimiento

- El impacto fue valorado de la siguiente forma:

- Alto: con una valoración de 3

- Medio: con una valoración de 2

- Bajo: con una valoración de 1

Para verificar el criterio de valoración y calificación de la matriz PEST-LA, se elaboró una tabla de variables por cada elemento, analizado los factores; político, económico, social-cultural, tecnológico, legal y ambiental de la provincia de Cotopaxi. Para identificar y diagnosticar los problemas que están obstaculizando el desarrollo del turismo sostenible en la provincia de Cotopaxi, se tomó en cuenta los elementos que no cumplen con la totalidad de variables planteadas en el criterio de valoración y calificación de la matriz PEST-LA, es decir, todos los elementos que han sido determinados con impacto negativo serán considerados como problemas turísticos de la provincia de Cotopaxi.

\section{Población y muestra}

El presente estudio se realizó en la provincia de Cotopaxi, se encuentra ubicada en el sector centro - norte del callejón andino, ocupa la Hoya Central Oriental del Patate. Se extiende desde los $78^{\circ}$ y $23^{\prime}$ en la parte oriental hasta los $79^{\circ}$ y $20^{\prime}$ en el sector occidental, en la Longitud de Greenwich; en sus 
extremos desde $0^{\circ}, 20^{\prime}$ hasta $1^{\circ}, 12^{\prime}$ de Latitud Sur. Se halla entre los 150 y los 5987 m.s.n.m. en los altos páramos de las cordilleras Occidental y Oriental, en la cual se encuentra el volcán activo más alto del mundo, el Cotopaxi con una elevación de 5897m.s.n.m. Limita al Norte con la provincia de Pichincha y Santo Domingo de los Tsáchilas; al Sur con las provincias de Tungurahua y Bolívar, al Este con la Provincia del Ñapo y al Oeste con las provincias de Santo Domingo de los Tsáchilas y Los Ríos. Su capital es la ciudad de Latacunga. Su extensión total de la provincia es de $5956 \mathrm{Km} 2$, con una densidad poblacional de 52 habitantes por $\mathrm{Km} 2$, que según el Censo de Población y Vivienda realizado en el año 2010 por el INEC, corresponde a una población total de 458.581 habitantes, de ellos el 58,2\% habitan en el sector rural y el $41,8 \%$ habitan en el área urbana, con el $49 \%$ de población masculina y el $51 \%$ femenina; distribuidos en los siete cantones (Latacunga, Pujilí, Salcedo, Saquisilí, La Maná, Pangua y Sigchos); 12 parroquias urbanas y 33 rurales (Muñoz \& Rodas, 2017).

La población de estudio fueron los 458.581 habitantes de los 7 cantones de la provincia de Cotopaxi; Latacunga, Saquisili, Pujili, Salcedo, La Mana, Pangua y Sigchos. El muestreo fue no probabilístico a conveniencia del equipo investigador, por motivos de distancia, población y desarrollo se seleccionaron actores claves del funcionamiento de los ambientes; político, económico, social-cultural, tecnológico, legal y ambiental de los cantones; Latacunga, Saquisili, Pujili, Salcedo y La Mana, y se aplicó el cuestionario validado por el equipo investigador (validez de contenido), a los actores clave seleccionados a través de entrevistas realizadas de manera aislada, en diferentes tiempos y espacios, con la finalidad de obtener información real y veraz.

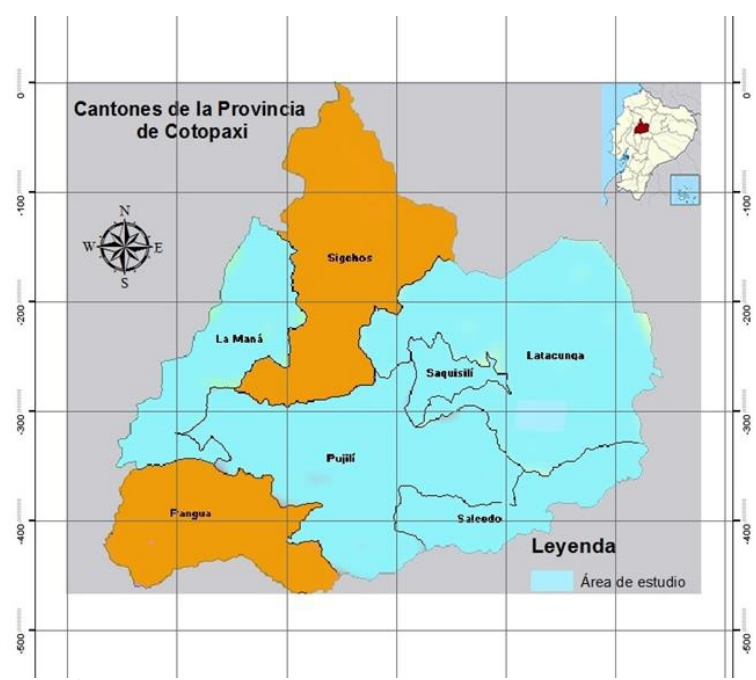

Figura 1. Área de estudio de la provincia de Cotopaxi, Ecuador.

Fuente: Adaptado de:

https://esacademic.com/pictures/eswiki/67/Cantones_de_la_Provincia_de_Cotopaxi.JPG 
Tabla 4. Población de los cantones en estudio de la provincia de Cotopaxi

\begin{tabular}{|c|c|c|}
\hline Cantón & $\underline{\text { Población }}$ & Parroquias \\
\hline$\overline{\text { Latacunga }}$ & 170.489 habitantes & $\begin{array}{c}\text { Distribuida en quince parroquias: } \\
\text { Diez Urbanas y } 5 \text { rurales }\end{array}$ \\
\hline Salcedo & 58.216 habitantes & $\begin{array}{c}\text { Constituida por } 6 \text { parroquias: } \\
1 \text { urbana y } 5 \text { rurales }\end{array}$ \\
\hline Pujilí & 69.055 habitantes & Distribuida en 7 parroquias \\
\hline Saquisilí & 25.320 habitantes & $\begin{array}{c}\text { Distribuida en } 4 \text { parroquias: } \\
1 \text { Urbana y } 3 \text { rurales }\end{array}$ \\
\hline La Mana & 49.035 habitantes & $\begin{array}{c}\text { Distribuida en } 5 \text { parroquias: } \\
3 \text { urbanas y } 2 \text { rurales. }\end{array}$ \\
\hline
\end{tabular}

Fuente: Adaptado del PDOT 2015-2025 de la provincia de Cotopaxi.

\section{3. $\quad$ Técnicas e instrumentos}

Se analizó información secundaria a través del llenado de fichas de revisión bibliográfica con base en la información de los siguientes documentos; Plan de Desarrollo y ordenamiento Territorial (PDOT) 20152025 , Plan de Desarrollo Turístico e Inventario de Atractivos Turísticos de la provincia de Cotopaxi, Plan de Desarrollo y ordenamiento Territorial (PDOT) 2016-2028 y catastro turístico del cantón Latacunga, Plan de Desarrollo y ordenamiento Territorial (PDOT) 2015-2020 y catastro turístico del cantón La Mana, Plan de Desarrollo y ordenamiento Territorial (PDOT) 2015-2020 y catastro turístico del cantón Salcedo, Plan de Desarrollo y ordenamiento Territorial (PDOT) 2014-2019 y catastro turístico del cantón Saquisilí, Plan de Desarrollo y ordenamiento Territorial (PDOT) 2015-2020 y catastro turístico del cantón Pujilí,

Se aplicó el cuestionario a través de entrevistas realizadas de manera aislada en diferentes tiempos y espacios, a los siguientes actores claves del funcionamiento de los ámbitos político, económico, social-cultural, tecnológico, legal y ambiental del área de estudio; 4 representantes del Gobierno Autónomo Descentralizado (GAD) de la provincia de Cotopaxi, 1 representante de cada uno de los 15 GADs parroquiales, 3 representantes de la cámara de turismo y 2 representantes de operadoras de turismo del cantón Latacunga. 1 Representante de cada uno de los 5 GADs parroquiales, 3 representantes de la cámara de turismo y 2 representantes de operadoras de turismo del cantón La Mana. 1 Representante de cada uno de los 6 GADs parroquiales, 3 representantes de la cámara de turismo y 2 representantes de operadoras de turismo cantón Salcedo. 1 Representante de cada uno de los 4 GADs parroquiales, 3 representantes de la cámara de turismo y 2 representantes de operadoras de turismo del cantón Saquisilí. 1 Representante de cada uno de los 7 GADs parroquiales, 3 representantes de la cámara de turismo y 2 representantes de operadoras de turismo del cantón Pujilí, 


\section{Resultados}

La matriz PEST-LA es una herramienta que generalmente es utilizada en la administración estratégica de una empresa, permite identificar los aspectos del macro-entorno, realiza una investigación detallada de los asuntos que más influirán en el desarrollo de su actividad o proyecto que se quiera impulsar, sin embargo esta matriz ha sido adaptada en el presente proyecto de investigación, para evaluar el funcionamiento de los elementos influyentes en el macro-entorno especialmente en los factores; político, económico, socialcultural, tecnológico, legal y ambiental de la provincia de Cotopaxi. A continuación se detallan los elementos analizados:

Tabla 5. Matriz PEST-LA de la provincia de Cotopaxi

\begin{tabular}{|c|c|c|c|c|}
\hline \multicolumn{5}{|l|}{ LA MANÁ } \\
\hline Factor & Elementos Analizados & Impacto +/- & $\begin{array}{l}\text { Valoración } \\
\text { del impacto }\end{array}$ & Calificación \\
\hline \multirow{4}{*}{ Político } & $\begin{array}{l}\text { Cambio de autoridades en GAD } \\
\text { Municipal y Parroquial del } \\
\text { cantón. }\end{array}$ & Positivo & Medio & 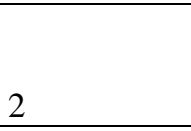 \\
\hline & $\begin{array}{l}\text { Coordinación entre agentes de } \\
\text { turismo y autoridades del } \\
\text { cantón para el desarrollo de } \\
\text { proyectos turísticos. }\end{array}$ & Negativo & Alto & 3 \\
\hline & $\begin{array}{l}\text { Promoción y difusión de los } \\
\text { atractivos turísticos del cantón } \\
\text { La Maná. }\end{array}$ & Negativo & Medio & 2 \\
\hline & $\begin{array}{l}\text { Infraestructura turística por } \\
\text { parte de las autoridades en los } \\
\text { sectores rurales del cantón. } \\
\end{array}$ & Negativo & Alto & 3 \\
\hline \multirow{3}{*}{ Económico } & $\begin{array}{l}\text { Crecimiento del segmento de } \\
\text { mercado nacional y extranjero } \\
\text { que visita el cantón. }\end{array}$ & Positivo & Alto & 3 \\
\hline & $\begin{array}{l}\text { Incremento de la planta turística } \\
\text { dentro del cantón. }\end{array}$ & Positivo & Alto & 3 \\
\hline & $\begin{array}{l}\text { Capacitación y planteamiento } \\
\text { de estrategias a proyectos } \\
\text { comunitarios que se sumen a la } \\
\text { actividad turística. }\end{array}$ & Negativo & Alto & 3 \\
\hline $\begin{array}{l}\text { Social- } \\
\text { Cultural }\end{array}$ & $\begin{array}{l}\text { Difusión y promoción de las } \\
\text { manifestaciones culturales que } \\
\text { se desarrollan en el cantón. }\end{array}$ & Negativo & Alto & 3 \\
\hline
\end{tabular}




\begin{tabular}{|c|c|c|c|c|}
\hline & $\begin{array}{l}\text { Adopción de otras culturas } \\
\text { provenientes de los turistas } \\
\text { (Alienación, a culturización) }\end{array}$ & Negativo & Alto & 3 \\
\hline & $\begin{array}{l}\text { Cambios en el estilo de vida de } \\
\text { los nativos por gustos y } \\
\text { preferencias de los turistas. }\end{array}$ & Positivo & Alto & 3 \\
\hline Tecnológico & $\begin{array}{l}\text { Cobertura de red telefónica } \\
\text { fija, móvil e internet. }\end{array}$ & Negativo & Alto & 3 \\
\hline \multirow{3}{*}{ Ambiental } & $\begin{array}{l}\text { Conciencia ambiental por parte } \\
\text { de los pobladores. }\end{array}$ & Negativo & Alto & 3 \\
\hline & Diversidad de flora y fauna. & Positivo & Alto & 3 \\
\hline & $\begin{array}{l}\text { Crecimiento de la frontera } \\
\text { agrícola y ganadera. }\end{array}$ & Negativo & Alto & 3 \\
\hline \multicolumn{5}{|l|}{ PUJILÍ } \\
\hline \multirow[b]{2}{*}{ Político } & $\begin{array}{l}\text { Interés en la ejecución de } \\
\text { proyectos. }\end{array}$ & Negativo & Medio & 2 \\
\hline & $\begin{array}{l}\text { Mantenimiento de la } \\
\text { infraestructura turística por } \\
\text { parte de las autoridades. }\end{array}$ & Negativo & Alto & 3 \\
\hline \multirow{4}{*}{ Económico } & $\begin{array}{l}\text { Presupuesto para ejecución de } \\
\text { proyectos. }\end{array}$ & Negativo & Medio & 2 \\
\hline & $\begin{array}{l}\text { Fuentes de empleos o empleos } \\
\text { mal remunerados }\end{array}$ & Negativo & Medio & 2 \\
\hline & $\begin{array}{l}\text { Población dedicada solo a un } \\
\text { ámbito laboral }\end{array}$ & Negativo & Alto & 3 \\
\hline & $\begin{array}{l}\text { Fondos destinados para la } \\
\text { ejecución de fiestas }\end{array}$ & Positivos & Alto & 3 \\
\hline \multirow{4}{*}{$\begin{array}{l}\text { Social- } \\
\text { Cultural }\end{array}$} & $\begin{array}{l}\text { Interés por las fiestas que tienen } \\
\text { alta relevancia en el cantón }\end{array}$ & Negativo & Medio & 2 \\
\hline & $\begin{array}{l}\text { Pérdida de valores y de } \\
\text { identidad cultural }\end{array}$ & Negativo & Alto & 3 \\
\hline & Creación de ferias artesanales & Positivo & Alto & 3 \\
\hline & Servicios médicos & Negativo & Medio & 2 \\
\hline \multirow{3}{*}{ Tecnológico } & $\begin{array}{l}\text { Implementación de páginas } \\
\text { web que dan a conocer las } \\
\text { distintas parroquias }\end{array}$ & Positivo & Medio & 2 \\
\hline & $\begin{array}{l}\text { Cobertura telefónica fija, móvil, } \\
\text { e internet }\end{array}$ & Negativo & Medio & 2 \\
\hline & $\begin{array}{l}\text { Interés por parte de la } \\
\text { población para conocer las } \\
\text { tendencias tecnológicas }\end{array}$ & Negativo & Alto & 3 \\
\hline Legal & $\begin{array}{l}\text { Limitación territorial de los } \\
\text { atractivos por parte de las } \\
\text { parroquias }\end{array}$ & Negativo & Medio & 2 \\
\hline
\end{tabular}




\begin{tabular}{|c|c|c|c|c|}
\hline & $\begin{array}{l}\text { Cumplimiento de las leyes } \\
\text { ambientales y turísticas }\end{array}$ & Negativo & Alto & 3 \\
\hline \multirow{6}{*}{ Ambiental } & $\begin{array}{l}\text { Conciencia ambiental por parte } \\
\text { de los pobladores. }\end{array}$ & Negativo & Alto & 3 \\
\hline & Manejo de desechos & Negativo & Alto & 3 \\
\hline & Servicio de alcantarillado & Negativo & Alto & 3 \\
\hline & Recolección de basura & Positivo & Medio & 2 \\
\hline & $\begin{array}{l}\text { Mantenimiento de atractivos } \\
\text { naturales }\end{array}$ & Negativo & Medio & 2 \\
\hline & $\begin{array}{l}\text { Conservación de los páramos } \\
\text { (frontera agrícola) }\end{array}$ & Negativo & Medio & 2 \\
\hline \multicolumn{5}{|c|}{ LATACUNGA } \\
\hline \multirow{5}{*}{ Político } & $\begin{array}{l}\text { Nuevos líderes en el sector } \\
\text { turístico }\end{array}$ & Positivo & Medio & 2 \\
\hline & $\begin{array}{l}\text { Políticas que regulen la } \\
\text { actividad turística }\end{array}$ & Negativo & Medio & 2 \\
\hline & Información turística & Negativo & Alto & 3 \\
\hline & $\begin{array}{l}\text { Elaboración de Planes, } \\
\text { programas y proyectos por } \\
\text { parte de entidades } \\
\text { gubernamentales sin ejecutarse }\end{array}$ & Negativo & Medio & 2 \\
\hline & $\begin{array}{l}\text { Programas y proyectos } \\
\text { gubernamentales (Plan Toda } \\
\text { una Vida) }\end{array}$ & Positivo & Alto & 3 \\
\hline \multirow{5}{*}{ Económico } & Incentivos para inversiones & Positivo & Medio & 2 \\
\hline & Gastronomía & Positivo & Alto & 3 \\
\hline & $\begin{array}{l}\text { Cercanía a atractivos } \\
\text { reconocidos a nivel } \\
\text { internacional } \\
\end{array}$ & Positivo & Alto & 3 \\
\hline & $\begin{array}{l}\text { Recursos económicos para } \\
\text { contratar personal }\end{array}$ & Negativo & Alto & 3 \\
\hline & $\begin{array}{l}\text { Actividad agrícola, florícola y } \\
\text { ganadera considerada como } \\
\text { fuente de ingreso económico }\end{array}$ & Negativo & Medio & 2 \\
\hline \multirow{7}{*}{$\begin{array}{l}\text { Social- } \\
\text { Cultural }\end{array}$} & $\begin{array}{l}\text { Sostenibilidad de recursos } \\
\text { culturales }\end{array}$ & Negativo & Medio & 2 \\
\hline & Interés en el desarrollo turístico & Negativo & Bajo & 1 \\
\hline & $\begin{array}{l}\text { Coordinación entre autoridades } \\
\text { y población }\end{array}$ & Negativo & Alto & 3 \\
\hline & Promoción de la oferta turística & Negativo & Medio & 2 \\
\hline & $\begin{array}{l}\text { Importancia hacia la actividad } \\
\text { agrícola }\end{array}$ & Negativo & Medio & 2 \\
\hline & \begin{tabular}{|l|} 
Competencia desleal entre los \\
prestadores de servicios
\end{tabular} & Negativo & Alto & 3 \\
\hline & $\begin{array}{l}\text { Empoderamiento del desarrollo } \\
\text { turístico por grandes empresas }\end{array}$ & Negativo & Alto & 3 \\
\hline
\end{tabular}




\begin{tabular}{|c|c|c|c|c|}
\hline \multirow{5}{*}{ Tecnológico } & $\begin{array}{l}\text { Implementación de Infocentros } \\
\text { (acceso a internet gratuito) }\end{array}$ & Positivo & Alto & 3 \\
\hline & $\begin{array}{l}\text { Aplicaciones móviles permiten } \\
\text { obtener información de un } \\
\text { destino turístico }\end{array}$ & Positivo & Medio & 2 \\
\hline & $\begin{array}{l}\text { Redes sociales permiten las } \\
\text { difusión de la información }\end{array}$ & Positivo & Medio & 2 \\
\hline & $\begin{array}{l}\text { Escaso conocimiento de nuevas } \\
\text { tecnologías }\end{array}$ & Negativo & Alto & 3 \\
\hline & Suficiente personal técnico & Positivo & Alto & 3 \\
\hline \multirow{4}{*}{ Legal } & $\begin{array}{l}\text { Incentivos a inversiones } \\
\text { privadas }\end{array}$ & Negativo & Medio & 2 \\
\hline & $\begin{array}{l}\text { Norma técnica de actividades } \\
\text { turísticas }\end{array}$ & Positivo & Medio & 2 \\
\hline & Norma técnica de Ecoturismo & Positivo & Medio & 2 \\
\hline & $\begin{array}{l}\text { Permisos para la apertura de } \\
\text { establecimientos turísticos }\end{array}$ & Negativo & Medio & 2 \\
\hline \multirow{6}{*}{ Ambiental } & $\begin{array}{l}\text { Inadecuado aprovechamiento } \\
\text { de los recursos (explotación } \\
\text { material pétreo) }\end{array}$ & Negativo & Alto & 3 \\
\hline & $\begin{array}{l}\text { Certificaciones turísticas } \\
\text { (no se ha obtenido suficientes) }\end{array}$ & Negativo & Medio & 2 \\
\hline & Proyectos de reciclaje & Positivo & Alto & 3 \\
\hline & Proyectos de reforestación & Positivo & Alto & 3 \\
\hline & $\begin{array}{l}\text { Sistema Nacional de Áreas } \\
\text { Protegidas }\end{array}$ & Positivo & Alto & 3 \\
\hline & $\begin{array}{l}\text { Organismos que protegen al } \\
\text { medio ambiente }\end{array}$ & Positivo & Alto & 3 \\
\hline \multicolumn{5}{|l|}{ SALCEDO } \\
\hline \multirow{5}{*}{ Político } & $\begin{array}{l}\text { Nuevos líderes en el sector } \\
\text { turístico }\end{array}$ & Positivo & Medio & 2 \\
\hline & $\begin{array}{l}\text { Políticas que regulen la } \\
\text { actividad turística }\end{array}$ & Negativo & Medio & 2 \\
\hline & Información turística & Negativo & Alto & 3 \\
\hline & $\begin{array}{l}\text { Elaboración de Planes, } \\
\text { programas y proyectos por } \\
\text { parte de entidades } \\
\text { gubernamentales sin ejecutarse }\end{array}$ & Negativo & Medio & 2 \\
\hline & $\begin{array}{l}\text { Programas y proyectos } \\
\text { gubernamentales (Plan Toda } \\
\text { una Vida) }\end{array}$ & Positivo & Alto & 3 \\
\hline \multirow{3}{*}{ Económico } & Incentivos para inversiones & Positivo & Medio & 2 \\
\hline & $\begin{array}{l}\text { Cantón reconocido por su } \\
\text { gastronomía }\end{array}$ & Positivo & Alto & 3 \\
\hline & $\begin{array}{l}\text { Cercanía a atractivos } \\
\text { reconocidos a nivel } \\
\text { internacional }\end{array}$ & Positivo & Alto & 3 \\
\hline
\end{tabular}




\begin{tabular}{|c|c|c|c|c|}
\hline & $\begin{array}{l}\text { Recursos económicos para } \\
\text { contratar personal }\end{array}$ & Negativo & Alto & 3 \\
\hline & $\begin{array}{l}\text { Actividad agrícola, florícola y } \\
\text { ganadera considerada como } \\
\text { fuente de ingreso económico }\end{array}$ & Negativo & Medio & 2 \\
\hline \multirow{7}{*}{ Social } & $\begin{array}{l}\text { Estado de los recursos } \\
\text { culturales }\end{array}$ & Negativo & Medio & 2 \\
\hline & Interés en el desarrollo turístico & Negativo & Bajo & 1 \\
\hline & $\begin{array}{l}\text { Coordinación entre autoridades } \\
\text { y población }\end{array}$ & Negativo & Alto & 3 \\
\hline & Promoción turística & Negativo & Medio & 2 \\
\hline & $\begin{array}{l}\text { Importancia hacia la actividad } \\
\text { agrícola }\end{array}$ & Negativo & Medio & 2 \\
\hline & $\begin{array}{l}\text { Competencia desleal entre los } \\
\text { prestadores de servicios }\end{array}$ & Negativo & Alto & 3 \\
\hline & $\begin{array}{l}\text { Empoderamiento del desarrollo } \\
\text { turístico por grandes empresas }\end{array}$ & Negativo & Alto & 3 \\
\hline \multirow{5}{*}{ Tecnológico } & $\begin{array}{l}\text { Implementación de Infocentros } \\
\text { (acceso a internet gratuito) }\end{array}$ & Positivo & Alto & 3 \\
\hline & $\begin{array}{l}\text { Aplicaciones móviles permiten } \\
\text { obtener información de un } \\
\text { destino turístico }\end{array}$ & Positivo & Medio & 2 \\
\hline & $\begin{array}{l}\text { Redes sociales permiten las } \\
\text { difusión de la información }\end{array}$ & Positivo & Medio & 2 \\
\hline & $\begin{array}{l}\text { Escaso conocimiento de nuevas } \\
\text { tecnologías }\end{array}$ & Negativo & Alto & 3 \\
\hline & Suficiente personal técnico & Positivo & Alto & 3 \\
\hline \multirow{4}{*}{ Legal } & $\begin{array}{l}\text { Incentivos a inversiones } \\
\text { privadas }\end{array}$ & Negativo & Medio & 2 \\
\hline & $\begin{array}{l}\text { Norma técnica de actividades } \\
\text { turísticas }\end{array}$ & Positivo & Medio & 2 \\
\hline & Norma técnica de ecoturismo & Positivo & Medio & 2 \\
\hline & $\begin{array}{l}\text { Permisos para la apertura de } \\
\text { establecimientos turísticos }\end{array}$ & Negativo & Medio & 2 \\
\hline \multirow{5}{*}{ Ambiental } & $\begin{array}{l}\text { Inadecuado aprovechamiento } \\
\text { de los recursos (explotación } \\
\text { material pétreo) }\end{array}$ & Negativo & Alto & 3 \\
\hline & $\begin{array}{l}\text { Certificaciones turísticas (hay } \\
\text { gran exigencias) }\end{array}$ & Negativo & Medio & 2 \\
\hline & Proyectos de reciclaje & Positivo & Alto & 3 \\
\hline & Proyectos de reforestación & Positivo & Alto & 3 \\
\hline & $\begin{array}{l}\text { Sistema Nacional de Áreas } \\
\text { Protegidas }\end{array}$ & Positivo & Alto & 3 \\
\hline \multicolumn{5}{|l|}{ SAQUISILÍ } \\
\hline \multirow[t]{2}{*}{ Político } & $\begin{array}{l}\text { Cambio de autoridades en el } \\
\text { GAD Municipal y Parroquial } \\
\text { del cantón. }\end{array}$ & Negativo & Medio & 2 \\
\hline & $\begin{array}{l}\text { Catastro turístico } \\
\text { desactualizado }\end{array}$ & Negativo & Medio & 2 \\
\hline
\end{tabular}




\begin{tabular}{|c|c|c|c|c|}
\hline & $\begin{array}{l}\text { Plan de Desarrollo y } \\
\text { Ordenamiento Territorial }\end{array}$ & Positivo & Alto & 3 \\
\hline \multirow{3}{*}{ Económico } & $\begin{array}{l}\text { Iniciativas por parte de las } \\
\text { personas encargadas para la } \\
\text { creación de proyectos }\end{array}$ & Positivo & Alto & 3 \\
\hline & $\begin{array}{l}\text { Escaso financiamiento } \\
\text { económico para la ejecución de } \\
\text { proyectos turísticos. }\end{array}$ & Negativo & Medio & 2 \\
\hline & $\begin{array}{l}\text { Creación de estrategias para } \\
\text { que más comunidades se sumen } \\
\text { a la actividad turística. }\end{array}$ & Positivo & Medio & 2 \\
\hline \multirow{6}{*}{$\begin{array}{l}\text { Socio- } \\
\text { Cultural }\end{array}$} & $\begin{array}{l}\text { El sector rural tiene déficit en } \\
\text { los servicios básicos }\end{array}$ & Negativo & Medio & 2 \\
\hline & Festividades Culturales & Positivo & Alto & 3 \\
\hline & Pérdida de identidad propia & Negativo & Medio & 2 \\
\hline & $\begin{array}{l}\text { Desinterés por parte de los } \\
\text { pobladores }\end{array}$ & Negativo & Medio & 2 \\
\hline & Señalización en los atractivos & Negativo & Alto & 3 \\
\hline & $\begin{array}{l}\text { Perdida de bienes } \\
\text { arquitectónicos }\end{array}$ & Negativo & Alto & 3 \\
\hline \multirow{3}{*}{ Tecnológico } & $\begin{array}{l}\text { El área rural no cuenta con } \\
\text { cobertura de red telefónica fija } \\
\text { y tampoco con el servicio de } \\
\text { internet }\end{array}$ & Negativo & Medio & 2 \\
\hline & $\begin{array}{l}\text { Implementación de infocentros } \\
\text { acceso a internet gratis en el } \\
\text { área urbana }\end{array}$ & Positivo & Alto & 3 \\
\hline & $\begin{array}{l}\text { Promoción mediante las } \\
\text { herramientas tecnológicas } \\
\text { actuales para la difusión de los } \\
\text { atractivos }\end{array}$ & Negativo & Medio & 2 \\
\hline Legal & $\begin{array}{l}\text { Intervención de las entidades } \\
\text { para la protección de los } \\
\text { páramos a través de ordenanzas }\end{array}$ & Negativo & Alto & 3 \\
\hline \multirow{3}{*}{ Ambiental } & $\begin{array}{l}\text { No todos los sitios turísticos } \\
\text { cuentan con el adecuado } \\
\text { manejo de residuos. }\end{array}$ & Negativo & Alto & 3 \\
\hline & $\begin{array}{l}\text { Baja conciencia ambiental por } \\
\text { parte de los pobladores. }\end{array}$ & Negativo & Alto & 3 \\
\hline & $\begin{array}{l}\text { Crecimiento de la frontera } \\
\text { agrícola y ganadera. }\end{array}$ & Negativo & Alto & 3 \\
\hline
\end{tabular}

Se analizaron 116 elementos, distribuidos en 5 factores; político, económico, social-cultural, tecnológico y ambiental de los cantones; La Mana, Salcedo, Saquisilí, Latacunga y Pujilí de la provincia de Cotopaxi. Se menciona que para valorar y calificar los elementos analizados, se elaboró una tabla de variables por cada elemento, analizando a detalle cada variable según 
evidencias encontradas, tanto a través de información segundaria como de información primaria. De los 116 elementos analizados; 42 fueron valorados como positivos y 74 fueron valorados como negativos, significando los elementos positivos analizados el $36 \%$ y los elementos negativos analizados el $64 \%$.

Para identificar y diagnosticar los problemas que están obstaculizando el desarrollo del turismo sostenible en la provincia de Cotopaxi, se tomó en cuenta los elementos que no cumplen con la totalidad de variables planteadas en el criterio de valoración y calificación de la matriz PEST-LA, es decir, todos los elementos que han sido determinados con impacto negativo serán considerados como problemas turísticos de la provincia de Cotopaxi. En consecuencia al criterio expuesto anteriormente en la tabla 5 se observa claramente que, de 116 elementos analizados 74 elementos fueron valorados como negativos, siendo elementos problema para el desarrollo del turismo en la provincia de Cotopaxi.

\section{Conclusiones}

Siendo la matriz PEST-LA una herramienta utilizada normalmente para analizar el macro-entorno de una organización, fue adaptada y utilizada para diagnosticar el funcionamiento de los factores; político, económico, social-cultural, tecnológico, legal y ambiental de los cantones; La Mana, Salcedo, Saquisilí, Latacunga y Pujilí de la provincia de Cotopaxi. Obteniendo como resultado: La Mana 9 elementos Negativos y 5 elementos positivos. Pujilì 16 elementos negativos y 3 positivos. Latacunga 17 elementos negativos y 15 positivos. Salcedo 17 elementos negativos y 15 positivos. Saquisili 14 elemntos negativos y 5 elementos positivos para el desarrollo turístico de la Provincia de Cotopaxi.

En conclusión, se analizaron 116 elementos fundamentales para el funcionamiento del macro-entorno turístico de la provincia de Cotopaxi. De los 116 elementos analizados, 42 fueron valorados como positivos y 74 elementos fueron valorados como negativos, siendo estos últimos considerados como problemas turísticos de la provincia, representando el 64 $\%$ de los elementos analizados en la presente investigación.

Según los 116 elementos analizados, se infiere que los principales problema del macro-entorno turístico de la provincia de Cotopaxi son; la adopción de otras culturas de vida de los nativos (a culturización-alienación), el crecimiento de la frontera agrícola, la deficiente coordinación entre agentes del turismo y autoridades, la falta de cobertura tecnológica y la dedicación a un solo ámbito laboral. Además los cantones con mayor problemas turísticos son; Pujilí y Saquisilí. 


\section{Recomendaciones}

Utilizar la matriz PEST-LA como una herramienta óptima para analizar el marco-entorno de un destino turístico, con base en el correcto funcionamiento de los factores; político, económico, social-cultural, tecnológico y ambiental del destino, como si fuera una organización a gran escala.

Las autoridades y actores claves del desarrollo turístico de la provincia de Cotopaxi (público, privado y comunitario), deben emprender un plan de mejoras para revertir de negativo a positivo, los 74 elementos que fueron valorados como problemas turísticos de la provincia, siendo estos el $68 \%$ de los elementos analizados en la presente investigación.

\section{References:}

1. Arteaga Basurto, C, \& M.V. Gonzáles Montaño. (2001). Diagnóstico. Obtenido de Desarrollo Comunitario: http://trabajosocialmazatlan.com/multimedia/files/InvestigacionPosgr ado/Diagnostico\%20Carlos\%20Arteaga.pdf

2. Bermúdez, F. (Mayo de 2002). GENERALIDADES DEL TURISMO . Obtenido de http://www.ina.ac.cr/documentos/turismo_aventura/GENERALIDA DES\%20DEL\%20TURISMO.pdf

3. Castillo, E., Herrera, G., \& Zambrano, D. (2016). IMPACTO ECONÓMICO DEL TURISMO EN LA ECONOMÍA DEL ECUADOR. Obtenido de https://abet.ufjf.emnuvens.com.br/abet/article/viewFile/2956/2260

4. Castillo, E., Martínez, F., \& Vázquez, E. (2015). EL TURISMO EN ECUADOR. NUEVAS TENDENCIAS EN EL TURISMO SOSTENIBLE Y CONTRIBUCIÓN AL CRECIMIENTO ECONÓMICO. Galega de Economía , 14-20.

5. Castillo, E., Vásquez, E., \& Martínez, F. (2015). Impacto Económico del Sector Turismo de Ecuador y UNASUR (1995 - 2013). Obtenido de

https://old.reunionesdeestudiosregionales.org/Reus2015/htdocs/pdf/p 1517.pdf

6. Cervantes, O. A. (2015). Administración Estratégica.

7. CFN. (2017). FICHA SECTORIAL: Sector Turístico- Nivel Nacional, Alojamiento y servicios de comida. Obtenido de https://www.cfn.fin.ec/wp-content/uploads/2017/10/Ficha-SectorialTurismo.pdf

8. Dalles, L. P. (30 de octubre de 2012). EL TURISMO II. Obtenido de EL TURISMO II: http://www.abc.com.py/edicionimpresa/suplementos/escolar/el-turismo-ii-471163.html 
9. Marketing, d. d. (2000). analisis y estrategias de desarrollo. En e. s. marketing. Madrid.

10. Ministerio de Turismo . (2018). Ministerio de Turismo . Obtenido de Ingreso de divisas por turismo : https://www.turismo.gob.ec/ingresode-divisas-por-turismo-crece-en-46-durante-el-primer-trimestre-de2018/

11. Ministerio de Turismo. (2015). PROYECTO ECUADOR POTENCIA TURÍSTICA. Obtenido de https://www.turismo.gob.ec/wpcontent/uploads/2015/04/Documento-Proyecto-Ecuador-PotenciaTur\%C3\%ADstica.pdf

12. MINTUR. (2018). Obtenido de https://www.turismo.gob.ec/turismogenera-en-ecuador-405-820-mil-fuentes-de-trabajo/

13. OMT. (Lunes de Julio de 2008). Introducción al turismo. Obtenido de http://www.profesionalesdelturismo.cl/images/libros/manuales/Introd uccion_al_Turismo.pdf

14. Pérez, P. (2008). Diagnosticos y tipos. Obtenido de Definicion de Diagnóstico: http://definicion.de/diagnostico/

15. Prieto, M. (2011). Los estudios sobre turismo en Ecuador. En Espacios en disputa: el turismo en Ecuador (págs. 9-28). Quito : FLACSO.

16. Quijano, C. (2009). Manual para el Diagnóstico Turistíco Local. Obtenido de Guía para Planificadores: http://unrn.edu.ar/blogs/pt/files/2013/03/6-Ricaurte09-GuiaPlanTurisver-fichas-al-final-post-2007.pdf

17. Ramirez, D. (17 de enero de 2013). Problemas sociales en el Turismo. Obtenido de Problemas sociales en el Turismo: http://dayanitaramirez.blogspot.com/

18. Rodriguez , D., \& Granados, P. (22 de Julio de 2016). Diagnóstico de Competitividad del Turismo. Obtenido de http://www.scielo.org.co/pdf/rfce/v25n2/0121-6805-rfce-25-0200193.pdf

19. Troitiño Vinueza, M., \& Troitiño Torralba, L. (2016). Patrimonio y turismo: reflexión teórico-conceptual y una propuesta metodológica integradora aplicada al municipio de Carmona (Sevilla, España). Scripta Nova, 15. 